Mathematical Sciences and Applications

E-NOTES

MATHEMATICAL

SCIENCES

AND APPLICATIONS

E-NOTES

https://doi.org/10.36753/mathenot.892258

9 (4) 185-193 (2021) - Research Article

ISSN: 2147-6268

(C)MSAEN

\title{
Some Remarks on the Equalities of Predictors in Linear Mixed Models
}

\author{
Melike Yiğit*, Nesrin Güler and Melek Eriş Büyükkaya
}

\begin{abstract}
Consider a transformed linear mixed model (TLMM) obtained pre-multiplying a linear mixed model (LMM) $\mathcal{M}: \mathbf{y}=\mathbf{Z} \boldsymbol{\alpha}+\mathbf{R} \boldsymbol{\gamma}+\mathbf{e}$ by a given matrix. This work concerns the problem of the equalities of linear predictors under the considered two LMMs under general assumptions. We characterize the equalities between the best linear unbiased predictors (BLUPs) under the LMM and its TLMM by using various rank formulas of block matrices and elementary matrix operations.
\end{abstract}

Keywords: BLUP; equalities; linear mixed model; random vectors; transformed model.

AMS Subject Classification (2020): Primary: 62J05 ; Secondary: 62H12; 15A03.

${ }^{*}$ Corresponding author

\section{Introduction}

Throughout this study, the symbol $\mathbb{R}^{m \times n}$ denotes the set of all $m \times n$ real matrices. $\mathbf{A}^{\prime}, \mathbf{A}^{+}, \boldsymbol{r}(\mathbf{A})$ and $\mathcal{C}(\mathbf{A})$ stand for the transpose, the Moore-Penrose generalized inverse, the rank, and the column space of $\mathbf{A} \in \mathbb{R}^{m \times n}$, respectively. $\mathbf{I}_{m}$ refers the $m \times m$ identity matrix. Furthermore, $\mathbf{E}_{\mathbf{A}}=\mathbf{A}^{\perp}=\mathbf{I}_{m}-\mathbf{A} \mathbf{A}^{+}$represents the orthogonal projector of $\mathbf{A} \in \mathbb{R}^{m \times n}$.

A linear mixed model (LMM) containing both fixed and random effects is formulated by

$$
\mathcal{M}: \mathbf{y}=\mathbf{Z} \boldsymbol{\alpha}+\mathbf{R} \gamma+\mathbf{e}
$$

where $\boldsymbol{\alpha}$ is a fixed effect and $\gamma$ is a random effect. In statistical inferences of analysis requirements, LMMs may need to be transformed. Several transformation methods can be used such as linear transformation. By doing this, the transformed linear mixed model (TLMM) of $\mathcal{M}$ is expressed as

$$
\mathcal{T}: \mathbf{T y}=\mathbf{T Z} \alpha+\mathbf{T R} \gamma+\mathbf{T e},
$$

which is obtained pre-multiplying $\mathcal{M}$ by a matrix $\mathbf{T}$. In two LMMs $\mathcal{M}$ and $\mathcal{T}, \mathbf{y} \in \mathbb{R}^{n \times 1}$ is a vector of observable response variables, $\mathbf{Z} \in \mathbb{R}^{n \times k}, \mathbf{R} \in \mathbb{R}^{n \times p}$, and $\mathbf{T} \in \mathbb{R}^{m \times n}$ are known matrices of arbitrary rank, $\boldsymbol{\alpha} \in \mathbb{R}^{k \times 1}$ is a vector of fixed but unknown parameters, $\gamma \in \mathbb{R}^{p \times 1}$ is a vector of unobservable random effects, and $\mathbf{e} \in \mathbb{R}^{n \times 1}$ is 
an unobservable vector of random errors. We will make the following general assumptions on expectations and dispersion matrices of random vectors in considered models

$$
\mathrm{E}\left[\begin{array}{l}
\gamma \\
\mathbf{e}
\end{array}\right]=\mathbf{0} \text { and } \mathrm{D}\left[\begin{array}{l}
\gamma \\
\mathbf{e}
\end{array}\right]=\operatorname{cov}\left\{\left[\begin{array}{l}
\gamma \\
\mathbf{e}
\end{array}\right],\left[\begin{array}{l}
\gamma \\
\mathbf{e}
\end{array}\right]\right\}=\left[\begin{array}{ll}
\boldsymbol{\Sigma}_{1} & \boldsymbol{\Sigma}_{2} \\
\boldsymbol{\Sigma}_{3} & \boldsymbol{\Sigma}_{4}
\end{array}\right]:=\boldsymbol{\Sigma}
$$

where $\boldsymbol{\Sigma}_{i}$ are known and $\boldsymbol{\Sigma} \in \mathbb{R}^{(n+p) \times(n+p)}$ is a positive semi-definite matrix of arbitrary rank, $i=1, \ldots, 4$. Let $\mathbf{A}=\left[\begin{array}{ll}\mathbf{R}, \mathbf{I}_{n}\end{array}\right]$ and then

$$
\mathrm{E}(\mathbf{y})=\mathbf{Z} \boldsymbol{\alpha}, \quad \mathrm{D}(\mathbf{y})=\left[\begin{array}{ll}
\mathbf{R}, & \mathbf{I}_{n}
\end{array}\right] \boldsymbol{\Sigma}\left[\mathbf{R}, \mathbf{I}_{n}\right]^{\prime}=\mathbf{A} \boldsymbol{\Sigma} \mathbf{A}^{\prime} .
$$

Further, assume that $\mathcal{M}$ is consistent, i.e.,

$$
\mathbf{y} \in \mathcal{C}\left[\mathbf{Z}, \quad \mathbf{A} \boldsymbol{\Sigma} \mathbf{A}^{\prime}\right] \text { holds with probability } 1(\text { wp } 1),
$$

see, e.g., [16]. The consistency assumption of the transformed model $\mathcal{T}$ is provided with $\mathbf{T y} \in \mathcal{C}\left[\mathbf{T Z}, \quad \mathbf{T A} \mathbf{\Sigma} \mathbf{A}^{\prime} \mathbf{T}^{\prime}\right]$ wp 1. It is easy to see that TLMM is consistent under the assumption of consistency of LMM.

In this study, we investigate the relations between the models $\mathcal{M}$ and $\mathcal{T}$. In order to characterize predictors simultaneously under two LMMs $\mathcal{M}$ and $\mathcal{T}$, the following vector can be considered

$$
\mathbf{u}=\mathbf{J} \boldsymbol{\alpha}+\mathbf{G} \boldsymbol{\gamma}+\mathbf{H e}=\mathbf{J} \boldsymbol{\alpha}+\left[\begin{array}{ll}
\mathbf{G}, & \mathbf{H}
\end{array}\right]\left[\begin{array}{l}
\gamma \\
\mathbf{e}
\end{array}\right]
$$

for given $\mathbf{J} \in \mathbb{R}^{s \times k}, \mathbf{G} \in \mathbb{R}^{s \times p}$, and $\mathbf{H} \in \mathbb{R}^{s \times n}$. Let $\mathbf{B}=\left[\begin{array}{ll}\mathbf{G}, \quad \mathbf{H}\end{array}\right]$, from (1.3) and (1.4), we obtain

$$
\begin{gathered}
\mathrm{E}(\mathbf{u})=\mathbf{J} \boldsymbol{\alpha}, \quad \mathrm{D}(\mathbf{u})=\left[\begin{array}{ll}
\mathbf{G}, & \mathbf{H}
\end{array}\right] \boldsymbol{\Sigma}\left[\begin{array}{ll}
\mathbf{G}, & \mathbf{H}
\end{array}\right]^{\prime}=\mathbf{B} \boldsymbol{\Sigma} \mathbf{B}^{\prime}, \\
\operatorname{cov}(\mathbf{u}, \mathbf{y})=\left[\begin{array}{ll}
\mathbf{G}, & \mathbf{H}
\end{array}\right] \boldsymbol{\Sigma}\left[\begin{array}{ll}
\mathbf{R}, & \mathbf{I}_{n}
\end{array}\right]^{\prime}=\mathbf{B} \boldsymbol{\Sigma} \mathbf{A}^{\prime} .
\end{gathered}
$$

The predictability requirement of vector $\mathbf{u}$ under $\mathcal{M}$ is described as holding the inclusion

$$
\mathcal{C}\left(\mathbf{J}^{\prime}\right) \subseteq \mathcal{C}\left(\mathbf{Z}^{\prime}\right) .
$$

Let $\mathbf{u}$ be predictable under $\mathcal{M}$. If there exists $\mathbf{F y}$ such that

$$
\mathrm{D}(\mathbf{F y}-\mathbf{u})=\text { min subject to } \mathrm{E}(\mathbf{F} \mathbf{y}-\mathbf{u})=\mathbf{0}
$$

holds in the Löwner partial ordering, then the best linear unbiased predictor (BLUP) of $\mathbf{u}$ is defined as Fy and is denoted by $\mathbf{F y}=\operatorname{BLUP}_{\mathcal{M}}(\mathbf{u})=\operatorname{BLUP}_{\mathcal{M}}(\mathbf{J} \boldsymbol{\alpha}+\mathbf{G} \boldsymbol{\gamma}+\mathbf{H e})$, originated from [6]. If $\mathbf{G}=\mathbf{0}$ and $\mathbf{H}=\mathbf{0}, \mathbf{F y}$ corresponds the best linear unbiased estimator (BLUE) of $\mathbf{J} \boldsymbol{\alpha}$, denoted by BLUE $\mathrm{M}_{\mathcal{M}}(\mathbf{J} \boldsymbol{\alpha})$, under $\mathcal{M}$.

Although predictors under LMMs and their TLMMs have different properties, observable random vectors in TLMMs may contain enough information to predict unknown vectors under LMMs. Within this context, establishing the results on the relations between these models can be considered as one of the important issues among others in linear regression analysis; see, e.g., $[4,7,22,24]$. We may also refer to the following works on relations between predictors under different LMMs; $[2,8-10,12,25]$. The problems of relations between original LMMs and their TLMMs are also closely connected to the concept of linear sufficiency, which was first introduced by $[3,5]$, see, also [11].

In this study, considering comparison problem of predictors under LMMs and their TLMMs, we derive the results on the equality characterizations between the BLUPs under $\mathcal{M}$ and $\mathcal{T}$. In order to characterize relations between BLUPs, we establish the results for the equality of coefficient matrices in the expressions of BLUPs under these models. For that purpose, we use the following expression on equality of random vectors.

$$
\mathbf{F}_{1} \mathbf{b}=\mathbf{F}_{2} \mathbf{b} \text { holds definitely if } \mathbf{F}_{1}=\mathbf{F}_{2} \text { for a random vector } \mathbf{b} \text {. }
$$

(1.11) means directly to solve the matrix equation $\mathbf{F}_{1}=\mathbf{F}_{2}$. We note that there are several types of equalities between two linear predictions $\mathbf{F}_{1} \mathbf{b}$ and $\mathbf{F}_{2} \mathbf{b}$ of a random vector $\mathbf{b}$, for details see, e.g., [4]. These equalities are defined according to different criteria for random vectors from the statistical point of view and (1.11) is one of these equality criteria. If coefficient matrices $\mathbf{F}_{1}$ and $\mathbf{F}_{2}$ in (1.11) are not unique, then $\mathbf{F}_{1}=\mathbf{F}_{2}$ can be divided into following four possible situations

$$
\left\{\mathbf{F}_{1}\right\} \cap\left\{\mathbf{F}_{2}\right\} \neq \emptyset, \quad\left\{\mathbf{F}_{1}\right\} \subset\left\{\mathbf{F}_{2}\right\}, \quad\left\{\mathbf{F}_{1}\right\} \supset\left\{\mathbf{F}_{2}\right\}, \quad\left\{\mathbf{F}_{1}\right\}=\left\{\mathbf{F}_{2}\right\},
$$


where $\left\{\mathbf{F}_{1}\right\}$ and $\left\{\mathbf{F}_{2}\right\}$ stand for the collections of all solutions of the equations. In accordance with (1.12), the equality between $\mathbf{F}_{1} \mathbf{b}$ and $\mathbf{F}_{2} \mathbf{b}$ can be divided into similar situations to (1.12). Considering the situations in (1.12), we give a comprehensive investigation in theoretical point of view to comparison of the BLUPs under the model $\mathcal{M}$ and its transformed model $\mathcal{T}$ by using various rank formulas of block matrices and elementary matrix operations. Various rank formulas for partitioned matrices provide us effective tools for simplifying complicated matrix expressions composed by matrices and their Moore-Penrose generalized inverses. The rank of matrices are one of the basic concepts in linear algebra and matrix theory, and also plays an essential role in problems on establishing equalities and inequalities occurred in statistical analysis; see, e.g., $[4,7,17,26]$.

\section{Preliminary Results}

This section briefly reviews the well-known results on linear matrix equations, some rank formulas of matrices, and the fundamental results on BLUP equations of $\mathbf{u}$ and related properties under models $\mathcal{M}$ and $\mathcal{T}$ that we will need for main results. The following lemma is given by [14].

Lemma 2.1. The linear matrix equation $\mathbf{M Z}=\mathbf{N}$ is consistent $\Leftrightarrow \boldsymbol{r}[\mathbf{M}, \quad \mathbf{N}]=\boldsymbol{r}(\mathbf{M})$, or equivalently, $\mathbf{M M}^{+} \mathbf{N}=\mathbf{N}$. In this case, the general solution of $\mathbf{M Z}=\mathbf{N}$ can be written as

$$
\mathbf{Z}=\mathbf{M}^{+} \mathbf{N}+\left(\mathbf{I}-\mathbf{M}^{+} \mathbf{M}\right) \mathbf{U}
$$

where $\mathbf{U}$ is an arbitrary matrix.

Let $\mathbf{u}$ in (1.6) be predictable under $\mathcal{M}$, i.e., (1.9) holds. Note that (1.10) is in fact a quadratic matrix optimization problem. The constrained covariance matrix minimization problem in (1.10) corresponds to a well-known fundamental BLUP equation, i.e.,

$$
\mathrm{E}(\mathbf{F} \mathbf{y}-\mathbf{u})=\mathbf{0} \text { and } \mathrm{D}(\mathbf{F y}-\mathbf{u})=\min \Leftrightarrow \mathbf{F}\left[\begin{array}{ll}
\mathbf{Z}, & \mathbf{A} \boldsymbol{\Sigma} \mathbf{A}^{\prime} \mathbf{Z}^{\perp}
\end{array}\right]=\left[\begin{array}{ll}
\mathbf{J}, & \mathbf{B} \boldsymbol{\Sigma} \mathbf{A}^{\prime} \mathbf{Z}^{\perp}
\end{array}\right] .
$$

According to Lemma 2.1, the general solution of (2.1) is written as

$$
\mathbf{F}=\left[\begin{array}{ll}
\mathbf{J}, & \mathbf{B} \Sigma \mathbf{A}^{\prime} \mathbf{Z}^{\perp}
\end{array}\right]\left[\begin{array}{ll}
\mathbf{Z}, & \mathbf{A} \Sigma \mathbf{A}^{\prime} \mathbf{Z}^{\perp}
\end{array}\right]^{+}+\mathbf{U}\left[\mathbf{Z}, \quad \mathbf{A} \Sigma \mathbf{A}^{\prime} \mathbf{Z}^{\perp}\right]^{\perp},
$$

where $\mathbf{U} \in \mathbb{R}^{s \times n}$ is an arbitrary matrix, and the BLUP of $\mathbf{u}$ under $\mathcal{M}$ is written as $\operatorname{BLUP}_{\mathcal{M}}(\mathbf{u})=\mathbf{F y}$ from (1.10). Further, we can add the following obvious results related to (2.1) and (2.2).

(a) The equation in (2.1) is always consistent.

(b) $\mathbf{F}$ in (2.2) is unique $\Leftrightarrow \boldsymbol{r}\left[\mathbf{Z}, \quad \mathbf{A} \boldsymbol{\Sigma} \mathbf{A}^{\prime} \mathbf{Z}^{\perp}\right]=n$.

(c) $\operatorname{BLUP}_{\mathcal{M}}(\mathbf{u})$ is unique wp $1 \Leftrightarrow \mathcal{M}$ is consistent, i.e., (1.5) holds.

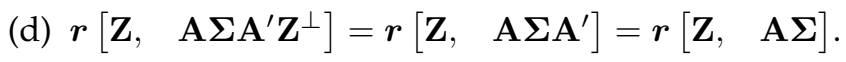

see, e.g., $[15,20,21]$.

Let us consider TLMM $\mathcal{T}$. The predictability requirement of $\mathbf{u}$ under $\mathcal{T}$ is expressed as

$$
\mathcal{C}\left(\mathbf{J}^{\prime}\right) \subseteq \mathcal{C}\left(\mathbf{Z}^{\prime} \mathbf{T}^{\prime}\right)
$$

It is evident that the predictability of $\mathbf{u}$ under a TLMM shows predictability of $\mathbf{u}$ under an original LMM. Let $\mathbf{u}$ in (1.6) be predictable under $\mathcal{T}$. The expression in (1.10) and the equation in (2.1) can be adapted for model $\mathcal{T}$ and thereby the fundamental BLUP equation under $\mathcal{T}$ is written as:

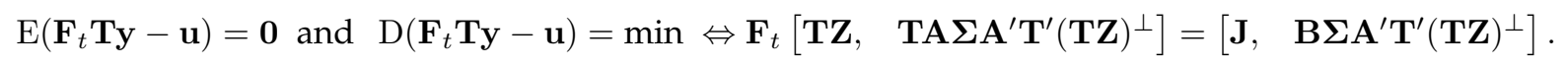

The matrix equation in (2.4) is always consistent. According to Lemma 2.1, the general solution of (2.4) is written as

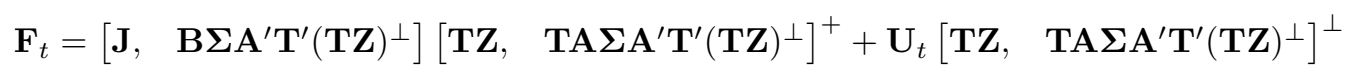

where $\mathbf{U}_{t} \in \mathbb{R}^{s \times m}$ is an arbitrary matrix, and $\operatorname{BLUP}_{\mathcal{T}}(\mathbf{u})=\mathbf{F}_{t} \mathbf{T y}$. Further, the expressions in (b)-(d) above for model $\mathcal{M}$ can be similarly expressed for model $\mathcal{T}$. 
The requirement in (1.9) corresponds to the estimability of vector $\mathbf{J} \boldsymbol{\alpha}$ under $\mathcal{M}$; see, e.g., [1], and, similarly, the requirement in (2.3) corresponds to the estimability of vector $\mathbf{J} \boldsymbol{\alpha}$ under $\mathcal{T}$. We also note that the estimability of vector $\mathbf{Z} \boldsymbol{\alpha}$ under both the models $\mathcal{M}$ and $\mathcal{T}$ is

$$
\boldsymbol{r}(\mathbf{Z})=\boldsymbol{r}(\mathbf{T Z}) .
$$

Let $\mathbf{J} \boldsymbol{\alpha}$ be estimable under $\mathcal{T}$ (also estimable under $\mathcal{M}$ ). The BLUEs of $\mathbf{J} \boldsymbol{\alpha}$ under models $\mathcal{M}$ and $\mathcal{T}$ are expressed as $\operatorname{BLUE}_{\mathcal{M}}(\mathbf{J} \boldsymbol{\alpha})=\mathbf{F}_{\mathbf{J} \boldsymbol{\alpha}} \mathbf{y}$ and $\operatorname{BLUE}_{\mathcal{T}}(\mathbf{J} \boldsymbol{\alpha})=\mathbf{F}_{t \mathbf{J} \boldsymbol{\alpha}} \mathbf{T} \mathbf{y}$, respectively, where

$$
\mathbf{F}_{\mathbf{J} \boldsymbol{\alpha}}=\left[\begin{array}{ll}
\mathbf{J}, & \mathbf{0}
\end{array}\right]\left[\begin{array}{ll}
\mathbf{Z}, & \mathbf{A} \boldsymbol{\Sigma} \mathbf{A}^{\prime} \mathbf{Z}^{\perp}
\end{array}\right]^{+}+\mathbf{U}\left[\mathbf{Z}, \quad \mathbf{A} \boldsymbol{\Sigma} \mathbf{A}^{\prime} \mathbf{Z}^{\perp}\right]^{\perp}
$$

and

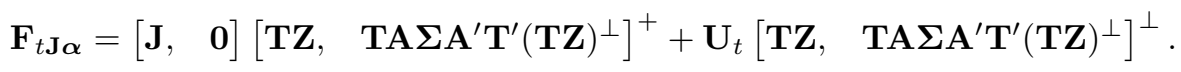

Let $\mathbf{Z} \boldsymbol{\alpha}$ be estimable under $\mathcal{T}$ (also estimable under $\mathcal{M}$ ). The BLUEs of $\mathbf{Z} \boldsymbol{\alpha}$ under models $\mathcal{M}$ and $\mathcal{T}$ are expressed as $\mathrm{BLUE}_{\mathcal{M}}(\mathbf{Z} \boldsymbol{\alpha})=\mathbf{F}_{\mathbf{Z} \boldsymbol{\alpha}} \mathbf{y}$ and $\mathrm{BLUE}_{\mathcal{T}}(\mathbf{Z} \boldsymbol{\alpha})=\mathbf{F}_{t \mathbf{Z} \boldsymbol{\alpha}} \mathbf{T y}$, respectively, where

$$
\mathbf{F}_{\mathbf{Z} \boldsymbol{\alpha}}=\left[\begin{array}{ll}
\mathbf{Z}, & \mathbf{0}
\end{array}\right]\left[\begin{array}{ll}
\mathbf{Z}, & \mathbf{A} \boldsymbol{\Sigma} \mathbf{A}^{\prime} \mathbf{Z}^{\perp}
\end{array}\right]^{+}+\mathbf{U}\left[\begin{array}{ll}
\mathbf{Z}, & \mathbf{A} \boldsymbol{\Sigma} \mathbf{A}^{\prime} \mathbf{Z}^{\perp}
\end{array}\right]^{\perp}
$$

and

$$
\mathbf{F}_{t \mathbf{Z} \alpha}=\left[\begin{array}{ll}
\mathbf{Z}, & \mathbf{0}
\end{array}\right]\left[\mathbf{T Z}, \quad \mathbf{T A} \mathbf{\Sigma} \mathbf{A}^{\prime} \mathbf{T}^{\prime}(\mathbf{T Z})^{\perp}\right]^{+}+\mathbf{U}_{t}\left[\mathbf{T Z}, \quad \mathbf{T A} \mathbf{\Sigma} \mathbf{A}^{\prime} \mathbf{T}^{\prime}(\mathbf{T Z})^{\perp}\right]^{\perp} .
$$

The following lemma is related to the characterizations in (1.12) based on (1.11); see, [19].

Lemma 2.2. Let $\mathbf{M} \in \mathbb{R}^{m \times n_{1}}, \mathbf{N} \in \mathbb{R}^{p \times n_{1}}, \mathbf{P} \in \mathbb{R}^{m \times n_{2}}$, and $\mathbf{Q} \in \mathbb{R}^{p \times n_{2}}$ be given. Then,

(a) Matrix equations $\mathbf{Z M}=\mathbf{N}$ and $\mathbf{Z P}=\mathbf{Q}$ have a common solution $\Leftrightarrow \mathcal{C}\left[\begin{array}{l}\mathbf{N}^{\prime} \\ \mathbf{Q}^{\prime}\end{array}\right] \subseteq \mathcal{C}\left[\begin{array}{l}\mathbf{M}^{\prime} \\ \mathbf{P}^{\prime}\end{array}\right] \Leftrightarrow \boldsymbol{r}\left[\begin{array}{ll}\mathbf{M} & \mathbf{P} \\ \mathbf{N} & \mathbf{Q}\end{array}\right]=$ $\boldsymbol{r}[\mathbf{M}, \mathbf{P}]$.

(b) Any solution of the matrix equation $\mathbf{Z P}=\mathbf{Q}$ is a solution of $\mathbf{Z M}=\mathbf{N} \Leftrightarrow \boldsymbol{r}\left[\begin{array}{ll}\mathbf{M} & \mathbf{P} \\ \mathbf{N} & \mathbf{Q}\end{array}\right]=\boldsymbol{r}(\mathbf{P})$.

In matrix algebra, some formulas of ranks of matrices are very helpful for facilitating complicated matrix equations. Within this framework, we use the following rank equalities for partitioned matrices; see [13].

Lemma 2.3. Let $\mathbf{M} \in \mathbb{R}^{m \times n}, \mathbf{N} \in \mathbb{R}^{m \times k}$, and $\mathbf{P} \in \mathbb{R}^{l \times n}$. Then,

$$
\begin{gathered}
r\left[\begin{array}{ll}
\mathbf{M}, & \mathbf{N}
\end{array}\right]=\boldsymbol{r}(\mathbf{M})+\boldsymbol{r}\left(\mathbf{E}_{\mathbf{M}} \mathbf{N}\right)=\boldsymbol{r}(\mathbf{N})+\boldsymbol{r}\left(\mathbf{E}_{\mathbf{N}} \mathbf{M}\right), \\
r\left[\begin{array}{c}
\mathbf{M} \\
\mathbf{P}
\end{array}\right]=\boldsymbol{r}(\mathbf{M})+\boldsymbol{r}\left(\mathbf{P E}_{\mathbf{M}^{\prime}}\right)=r(\mathbf{P})+\boldsymbol{r}\left(\mathbf{M E}_{\mathbf{P}^{\prime}}\right) .
\end{gathered}
$$

\section{Equality Relations of BLUPs under LMM and its TLMM}

In this section, the main results on the equalities between BLUPs, related to the characterizations in (1.12), under models $\mathcal{M}$ and $\mathcal{T}$ are given .

Theorem 3.1. Let us consider $\mathcal{M}$ in (1.1) and $\mathcal{T}$ in (1.2). Assume that $\mathbf{u}$ in (1.6) is predictable under these models. Let the coefficients $\mathbf{F}$ and $\mathbf{F}_{t}$ be as given in (2.2) and (2.5), respectively. Then

$$
\{\mathbf{F}\} \cap\left\{\mathbf{F}_{t} \mathbf{T}\right\} \neq \emptyset \Leftrightarrow \boldsymbol{r}\left[\begin{array}{ccccc}
\mathbf{A} \Sigma \mathbf{A}^{\prime} & \mathbf{0} & \mathbf{Z} & \mathbf{0} & \mathbf{I}_{n} \\
\mathbf{0} & \mathbf{T A} \mathbf{\Sigma} \mathbf{A}^{\prime} \mathbf{T}^{\prime} & \mathbf{0} & \mathbf{T Z} & \mathbf{T} \\
\mathbf{Z}^{\prime} & \mathbf{0} & \mathbf{0} & \mathbf{0} & \mathbf{0} \\
\mathbf{0} & \mathbf{Z}^{\prime} \mathbf{T}^{\prime} & \mathbf{0} & \mathbf{0} & \mathbf{0} \\
-\mathbf{B} \Sigma \mathbf{A}^{\prime} & \mathbf{B} \Sigma \mathbf{A}^{\prime} \mathbf{T}^{\prime} & -\mathbf{J} & \mathbf{J} & \mathbf{0}
\end{array}\right]=\boldsymbol{r}\left[\begin{array}{ccccc}
\mathbf{A} \Sigma \mathbf{A}^{\prime} & \mathbf{0} & \mathbf{Z} & \mathbf{0} & \mathbf{I}_{n} \\
\mathbf{0} & \mathbf{T A} \mathbf{\Sigma} \mathbf{A}^{\prime} \mathbf{T}^{\prime} & \mathbf{0} & \mathbf{T Z} & \mathbf{T} \\
\mathbf{Z}^{\prime} & \mathbf{0} & \mathbf{0} & \mathbf{0} & \mathbf{0} \\
\mathbf{0} & \mathbf{Z}^{\prime} \mathbf{T}^{\prime} & \mathbf{0} & \mathbf{0} & \mathbf{0}
\end{array}\right] .
$$

In this case, $\left\{\operatorname{BLUP}_{\mathcal{M}}(\mathbf{u})\right\} \cap\left\{\operatorname{BLUP}_{\mathcal{T}}(\mathbf{u})\right\} \neq \emptyset$ holds definitely. 
Proof. From (2.2) and (2.5), $\mathbf{F}-\mathbf{F}_{t} \mathbf{T}$ is written as

$$
\left[\mathbf{J}, \quad \mathbf{B} \boldsymbol{\Sigma} \mathbf{A}^{\prime} \mathbf{Z}^{\perp}\right] \mathbf{W}^{+}-\left[\mathbf{J}, \quad \mathbf{B} \boldsymbol{\Sigma} \mathbf{A}^{\prime} \mathbf{T}^{\prime}(\mathbf{T Z})^{\perp}\right] \mathbf{W}_{t}^{+} \mathbf{T}+\mathbf{U W}^{\perp}-\mathbf{U}_{t} \mathbf{W}_{t}^{\perp} \mathbf{T}
$$

where $\mathbf{W}=\left[\begin{array}{ll}\mathbf{Z}, & \mathbf{A} \boldsymbol{\Sigma} \mathbf{A}^{\prime} \mathbf{Z}^{\perp}\end{array}\right]$ and $\mathbf{W}_{t}=\left[\begin{array}{ll}\mathbf{T Z}, & \mathbf{T A} \mathbf{\Sigma} \mathbf{A}^{\prime} \mathbf{T}^{\prime}(\mathbf{T Z})^{\perp}\end{array}\right]$. Then applying the formula $\min _{\mathbf{U}} \boldsymbol{r}(\mathbf{C}+\mathbf{U D})=$ $\boldsymbol{r}\left[\begin{array}{l}\mathbf{C} \\ \mathbf{D}\end{array}\right]-\boldsymbol{r}(\mathbf{D})$, given in [18] and [23], to (3.2) and simplifying the block matrices by Lemma 2.3, we obtain

$$
\begin{aligned}
& =\min _{\mathbf{U}, \mathbf{U}_{t}} \boldsymbol{r}\left(\left[\begin{array}{ll}
\mathbf{J}, & \mathbf{B} \boldsymbol{\Sigma} \mathbf{A}^{\prime} \mathbf{Z}^{\perp}
\end{array}\right] \mathbf{W}^{+}-\left[\begin{array}{ll}
\mathbf{J}, & \mathbf{B} \boldsymbol{\Sigma} \mathbf{A}^{\prime} \mathbf{T}^{\prime}(\mathbf{T Z})^{\perp}
\end{array}\right] \mathbf{W}_{t}^{+} \mathbf{T}+\left[\begin{array}{ll}
\mathbf{U}, & -\mathbf{U}_{t}
\end{array}\right]\left[\begin{array}{c}
\mathbf{W}^{\perp} \\
\mathbf{W}_{t}^{\perp} \mathbf{T}
\end{array}\right]\right) \\
& =\boldsymbol{r}\left[\begin{array}{cc}
\left.\left.\mathbf{J}, \quad \mathbf{B} \Sigma \mathbf{A}^{\prime} \mathbf{Z}^{\perp}\right] \mathbf{W}^{+}-\underset{\mathbf{W}^{\perp}}{[\mathbf{J},} \mathbf{B} \Sigma \mathbf{A}^{\prime} \mathbf{T}^{\prime}(\mathbf{T Z})^{\perp}\right] \mathbf{W}_{t}^{+} \mathbf{T} \\
\mathbf{W}_{t}^{\perp} \mathbf{T}
\end{array}\right]-\boldsymbol{r}\left[\begin{array}{c}
\mathbf{W}^{\perp} \\
\mathbf{W}_{t}^{\perp} \mathbf{T}
\end{array}\right]
\end{aligned}
$$

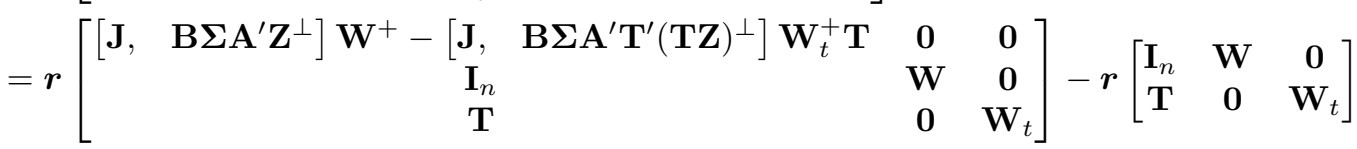

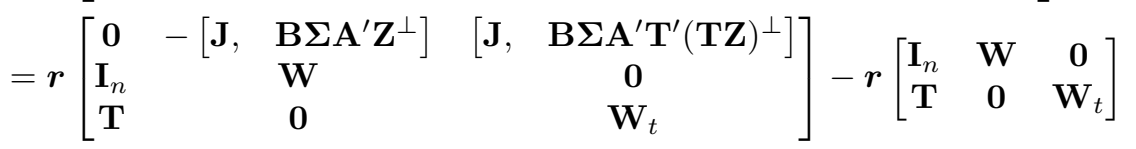

$$
\begin{aligned}
& =\boldsymbol{r}\left[\begin{array}{ccccc}
\mathbf{0} & -\mathbf{J} & -\mathbf{B} \boldsymbol{\Sigma} \mathbf{A}^{\prime} & \mathbf{J} & \mathbf{B} \boldsymbol{\Sigma} \mathbf{A}^{\prime} \mathbf{T}^{\prime} \\
\mathbf{I}_{n} & \mathbf{Z} & \mathbf{A} \boldsymbol{\Sigma} \mathbf{A}^{\prime} & \mathbf{0} & \mathbf{0} \\
\mathbf{T} & \mathbf{0} & \mathbf{0} & \mathbf{T Z} & \mathbf{T A} \mathbf{\Sigma} \mathbf{A}^{\prime} \mathbf{T}^{\prime} \\
\mathbf{0} & \mathbf{0} & \mathbf{Z}^{\prime} & \mathbf{0} & \mathbf{0} \\
\mathbf{0} & \mathbf{0} & \mathbf{0} & \mathbf{0} & \mathbf{Z}^{\prime} \mathbf{T}^{\prime}
\end{array}\right]-\boldsymbol{r}\left[\begin{array}{ccccc}
\mathbf{I}_{n} & \mathbf{Z} & \mathbf{A} \boldsymbol{\Sigma} \mathbf{A}^{\prime} & \mathbf{0} & \mathbf{0} \\
\mathbf{T} & \mathbf{0} & \mathbf{0} & \mathbf{T Z} & \mathbf{T A} \mathbf{\Sigma} \mathbf{A}^{\prime} \mathbf{T}^{\prime} \\
\mathbf{0} & \mathbf{0} & \mathbf{Z}^{\prime} & \mathbf{0} & \mathbf{0} \\
\mathbf{0} & \mathbf{0} & \mathbf{0} & \mathbf{0} & \mathbf{Z}^{\prime} \mathbf{T}^{\prime}
\end{array}\right] \\
& =\boldsymbol{r}\left[\begin{array}{ccccc}
\mathbf{A} \Sigma \mathbf{A}^{\prime} & \mathbf{0} & \mathbf{Z} & \mathbf{0} & \mathbf{I}_{n} \\
\mathbf{0} & \mathbf{T A} \Sigma \mathbf{A}^{\prime} \mathbf{T}^{\prime} & \mathbf{0} & \mathbf{T Z} & \mathbf{T} \\
\mathbf{Z}^{\prime} & \mathbf{0} & \mathbf{0} & \mathbf{0} & \mathbf{0} \\
\mathbf{0} & \mathbf{Z}^{\prime} \mathbf{T}^{\prime} & \mathbf{0} & \mathbf{0} & \mathbf{0} \\
-\mathbf{B} \Sigma \mathbf{A}^{\prime} & \mathbf{B} \Sigma \mathbf{A}^{\prime} \mathbf{T}^{\prime} & -\mathbf{J} & \mathbf{J} & \mathbf{0}
\end{array}\right]-\boldsymbol{r}\left[\begin{array}{ccccc}
\mathbf{A} \Sigma \mathbf{A}^{\prime} & \mathbf{0} & \mathbf{Z} & \mathbf{0} & \mathbf{I}_{n} \\
\mathbf{0} & \mathbf{T A} \mathbf{A} \mathbf{A}^{\prime} \mathbf{T}^{\prime} & \mathbf{0} & \mathbf{T Z} & \mathbf{T} \\
\mathbf{Z}^{\prime} & \mathbf{0} & \mathbf{0} & \mathbf{0} & \mathbf{0} \\
\mathbf{0} & \mathbf{Z}^{\prime} \mathbf{T}^{\prime} & \mathbf{0} & \mathbf{0} & \mathbf{0}
\end{array}\right] .
\end{aligned}
$$

The required result is seen from (3.3).

Corollary 3.1. Let us consider $\mathcal{M}$ in (1.1) and $\mathcal{T}$ in (1.2).

(a) Let $\mathbf{J} \boldsymbol{\alpha}$ be estimable under $\mathcal{T}$ (also estimable under $\mathcal{M}$ ). Let the coefficients $\mathbf{F}_{\mathbf{J} \boldsymbol{\alpha}}$ and $\mathbf{F}_{t \mathbf{J} \boldsymbol{\alpha}}$ be as given in (2.7) and (2.8), respectively. Then the following holds.

$$
\begin{aligned}
\left\{\mathbf{F}_{\mathbf{J} \boldsymbol{\alpha}}\right\} & \cap\left\{\mathbf{F}_{t \mathbf{J} \boldsymbol{\alpha}} \mathbf{T}\right\} \neq \emptyset \\
& \Leftrightarrow \boldsymbol{r}\left[\begin{array}{ccccc}
\mathbf{A} \mathbf{\Sigma} \mathbf{A}^{\prime} & \mathbf{0} & \mathbf{Z} & \mathbf{0} & \mathbf{I}_{n} \\
\mathbf{0} & \mathbf{T A} \boldsymbol{\Sigma} \mathbf{A}^{\prime} \mathbf{T}^{\prime} & \mathbf{0} & \mathbf{T Z} & \mathbf{T} \\
\mathbf{Z}^{\prime} & \mathbf{0} & \mathbf{0} & \mathbf{0} & \mathbf{0} \\
\mathbf{0} & \mathbf{Z}^{\prime} \mathbf{T}^{\prime} & \mathbf{0} & \mathbf{0} & \mathbf{0} \\
\mathbf{0} & \mathbf{0} & -\mathbf{J} & \mathbf{J} & \mathbf{0}
\end{array}\right]=\boldsymbol{r}\left[\begin{array}{ccccc}
\mathbf{A} \boldsymbol{\Sigma} \mathbf{A}^{\prime} & \mathbf{0} & \mathbf{Z} & \mathbf{0} & \mathbf{I}_{n} \\
\mathbf{0} & \mathbf{T A} \boldsymbol{\mathbf { \Sigma }} \mathbf{A}^{\prime} \mathbf{T}^{\prime} & \mathbf{0} & \mathbf{T Z} & \mathbf{T} \\
\mathbf{Z}^{\prime} & \mathbf{0} & \mathbf{0} & \mathbf{0} & \mathbf{0} \\
\mathbf{0} & \mathbf{Z}^{\prime} \mathbf{T}^{\prime} & \mathbf{0} & \mathbf{0} & \mathbf{0}
\end{array}\right] .
\end{aligned}
$$

In this case, $\left\{\operatorname{BLUE}_{\mathcal{M}}(\mathbf{J} \boldsymbol{\alpha})\right\} \cap\left\{\operatorname{BLUP}_{\mathcal{T}}(\mathbf{J} \boldsymbol{\alpha})\right\} \neq \emptyset$ holds definitely.

(b) If $\mathbf{Z} \boldsymbol{\alpha}$ is estimable under the models $\mathcal{M}$ and $\mathcal{T}$ then (2.6) holds. Let the coefficients $\mathbf{F}_{\mathbf{Z} \boldsymbol{\alpha}}$ and $\mathbf{F}_{t \mathbf{z} \boldsymbol{\alpha}}$ be as given in (2.9) and (2.10), respectively. Then the following holds.

$$
\left\{\mathbf{F}_{\mathbf{Z} \boldsymbol{\alpha}}\right\} \cap\left\{\mathbf{F}_{t \mathbf{Z} \boldsymbol{\alpha}} \mathbf{T}\right\} \neq \emptyset \Leftrightarrow \boldsymbol{r}\left[\begin{array}{ccc}
\mathbf{A} \boldsymbol{\Sigma} \mathbf{A}^{\prime} & \mathbf{0} & \mathbf{I}_{n} \\
\mathbf{0} & \mathbf{T A} \boldsymbol{\Sigma} \mathbf{A}^{\prime} \mathbf{T}^{\prime} & \mathbf{T} \\
\mathbf{Z}^{\prime} & \mathbf{0} & \mathbf{0} \\
\mathbf{0} & \mathbf{Z}^{\prime} \mathbf{T}^{\prime} & \mathbf{0}
\end{array}\right]=\boldsymbol{r}\left[\begin{array}{ccc}
\mathbf{A} \Sigma \mathbf{A}^{\prime} & \mathbf{Z} & \mathbf{I}_{n} \\
\mathbf{0} & \mathbf{0} & \mathbf{T}
\end{array}\right]+\boldsymbol{r}(\mathbf{Z}) .
$$

In this case, $\left\{\operatorname{BLUE}_{\mathcal{M}}(\mathbf{Z} \boldsymbol{\alpha})\right\} \cap\left\{\operatorname{BLUE}_{\mathcal{T}}(\mathbf{Z} \boldsymbol{\alpha})\right\} \neq \emptyset$. 
Theorem 3.2. Let us consider $\mathcal{M}$ in (1.1) and $\mathcal{T}$ in (1.2). Assume that $\mathbf{u}$ in (1.6) is predictable under these models. Let the coefficients $\mathbf{F}$ and $\mathbf{F}_{t}$ be as given in (2.2) and (2.5), respectively. Then

$$
\{\mathbf{F}\} \subset\left\{\mathbf{F}_{t} \mathbf{T}\right\} \Leftrightarrow \boldsymbol{r}\left[\begin{array}{ccc}
\mathbf{A} \Sigma \mathbf{A}^{\prime} & \mathbf{A} \boldsymbol{\Sigma} \mathbf{A}^{\prime} \mathbf{T}^{\prime} & \mathbf{Z} \\
\mathbf{Z}^{\prime} & \mathbf{0} & \mathbf{0} \\
\mathbf{0} & \mathbf{Z}^{\prime} \mathbf{T}^{\prime} & \mathbf{0} \\
\mathbf{B} \boldsymbol{\Sigma} \mathbf{A}^{\prime} & \mathbf{B} \Sigma \mathbf{A}^{\prime} \mathbf{T}^{\prime} & \mathbf{J}
\end{array}\right]=\boldsymbol{r}\left[\begin{array}{cc}
\mathbf{A} \Sigma \mathbf{A}^{\prime} & \mathbf{Z} \\
\mathbf{Z}^{\prime} & \mathbf{0}
\end{array}\right]+\boldsymbol{r}(\mathbf{Z}) .
$$

In this case, $\left\{\operatorname{BLUP}_{\mathcal{M}}(\mathbf{u})\right\} \subset\left\{\operatorname{BLUP}_{\mathcal{T}}(\mathbf{u})\right\}$ holds.

Proof. From Lemma 2.2 (b), all solutions of the equation in (2.1) are the solutions of the equation in (2.4) $\Leftrightarrow$

$$
\boldsymbol{r}\left[\begin{array}{llll}
\mathbf{Z} & \mathbf{A} \Sigma \mathbf{A}^{\prime} \mathbf{Z}^{\perp} & \mathbf{Z} & \mathbf{A} \Sigma \mathbf{A}^{\prime} \mathbf{T}^{\prime}(\mathbf{T Z})^{\perp} \\
\mathbf{J} & \mathbf{B} \Sigma \mathbf{A}^{\prime} \mathbf{Z}^{\perp} & \mathbf{J} & \mathbf{B} \Sigma \mathbf{A}^{\prime} \mathbf{T}^{\prime}(\mathbf{T Z})^{\perp}
\end{array}\right]=r\left[\mathbf{Z}, \quad \mathbf{A} \Sigma \mathbf{A}^{\prime} \mathbf{Z}^{\perp}\right]
$$

(3.7) equivalently written as

$$
\boldsymbol{r}\left[\begin{array}{cccc}
\mathbf{Z} & \mathbf{A} \Sigma \mathbf{A}^{\prime} & \mathbf{Z} & \mathbf{A} \boldsymbol{\Sigma} \mathbf{A}^{\prime} \mathbf{T}^{\prime} \\
\mathbf{J} & \mathbf{B} \Sigma \mathbf{A}^{\prime} & \mathbf{J} & \mathbf{B} \boldsymbol{\Sigma} \mathbf{A}^{\prime} \mathbf{T}^{\prime} \\
\mathbf{0} & \mathbf{Z}^{\prime} & \mathbf{0} & \mathbf{0} \\
\mathbf{0} & \mathbf{0} & \mathbf{0} & \mathbf{Z}^{\prime} \mathbf{T}^{\prime}
\end{array}\right]-\boldsymbol{r}(\mathbf{Z})-\boldsymbol{r}(\mathbf{T Z})=\boldsymbol{r}\left[\begin{array}{cc}
\mathbf{Z} & \mathbf{A} \boldsymbol{\Sigma} \mathbf{A}^{\prime} \\
\mathbf{0} & \mathbf{Z}^{\prime}
\end{array}\right]-\boldsymbol{r}(\mathbf{Z})
$$

which is equivalent to (3.6).

Corollary 3.2. Let us consider $\mathcal{M}$ in (1.1) and $\mathcal{T}$ in (1.2).

(a) Let $\mathbf{J} \boldsymbol{\alpha}$ be estimable under $\mathcal{T}$ (also estimable under $\mathcal{M}$ ). Let the coefficients $\mathbf{F}_{\mathbf{J} \boldsymbol{\alpha}}$ and $\mathbf{F}_{t \mathbf{J} \boldsymbol{\alpha}}$ be as given in (2.7) and (2.8), respectively. Then the following holds.

$$
\left\{\mathbf{F}_{\mathbf{J} \boldsymbol{\alpha}}\right\} \subset\left\{\mathbf{F}_{t \mathbf{J} \boldsymbol{\alpha}} \mathbf{T}\right\} \Leftrightarrow \boldsymbol{r}\left[\begin{array}{ccc}
\mathbf{A} \boldsymbol{\Sigma} \mathbf{A}^{\prime} & \mathbf{A} \boldsymbol{\Sigma} \mathbf{A}^{\prime} \mathbf{T}^{\prime} & \mathbf{Z} \\
\mathbf{Z}^{\prime} & \mathbf{0} & \mathbf{0} \\
\mathbf{0} & \mathbf{Z}^{\prime} \mathbf{T}^{\prime} & \mathbf{0} \\
\mathbf{0} & \mathbf{0} & \mathbf{J}
\end{array}\right]=\boldsymbol{r}\left[\begin{array}{cc}
\mathbf{A} \boldsymbol{\Sigma} \mathbf{A}^{\prime} & \mathbf{Z} \\
\mathbf{Z}^{\prime} & \mathbf{0}
\end{array}\right]+\boldsymbol{r}(\mathbf{Z}) .
$$

In this case, $\left\{\operatorname{BLUE}_{\mathcal{M}}(\mathbf{J} \boldsymbol{\alpha})\right\} \subset\left\{\operatorname{BLUE}_{\mathcal{T}}(\mathbf{J} \boldsymbol{\alpha})\right\}$ holds.

(b) If $\mathbf{Z} \boldsymbol{\alpha}$ is estimable under the models $\mathcal{M}$ and $\mathcal{T}$ then (2.6) holds. Let the coefficients $\mathbf{F}_{\mathbf{Z} \boldsymbol{\alpha}}$ and $\mathbf{F}_{t \mathbf{Z} \boldsymbol{\alpha}}$ be as given in (2.9) and (2.10), respectively. Then the following holds.

$$
\left\{\mathbf{F}_{\mathbf{Z} \boldsymbol{\alpha}}\right\} \subset\left\{\mathbf{F}_{t \mathbf{Z} \boldsymbol{\alpha}} \mathbf{T}\right\} \Leftrightarrow \boldsymbol{r}\left[\begin{array}{cc}
\mathbf{A} \boldsymbol{\Sigma} \mathbf{A}^{\prime} & \mathbf{A} \boldsymbol{\Sigma} \mathbf{A}^{\prime} \mathbf{T}^{\prime} \\
\mathbf{Z}^{\prime} & \mathbf{0} \\
\mathbf{0} & \mathbf{Z}^{\prime} \mathbf{T}^{\prime}
\end{array}\right]=\boldsymbol{r}\left[\begin{array}{ccc}
\mathbf{A} \boldsymbol{\Sigma} \mathbf{A}^{\prime} & \mathbf{Z} \\
\mathbf{Z}^{\prime} & \mathbf{0}
\end{array}\right] .
$$

In this case, $\left\{\operatorname{BLUE}_{\mathcal{M}}(\mathbf{Z} \boldsymbol{\alpha})\right\} \subset\left\{\operatorname{BLUE}_{\mathcal{T}}(\mathbf{Z} \boldsymbol{\alpha})\right\}$ holds.

Theorem 3.3. Let us consider $\mathcal{M}$ in (1.1) and $\mathcal{T}$ in (1.2). Assume that $\mathbf{u}$ in (1.6) is predictable under these models. Let the coefficients $\mathbf{F}$ and $\mathbf{F}_{t}$ be as given in (2.2) and (2.5), respectively. Then

$$
\mathbf{F} \in\left\{\mathbf{F}_{t} \mathbf{T}\right\} \Leftrightarrow \boldsymbol{r}\left[\begin{array}{ccc}
\mathbf{A} \boldsymbol{\Sigma} \mathbf{A}^{\prime} & \mathbf{A} \boldsymbol{\Sigma} \mathbf{A}^{\prime} \mathbf{T}^{\prime} & \mathbf{Z} \\
\mathbf{Z}^{\prime} & \mathbf{0} & \mathbf{0} \\
\mathbf{0} & \mathbf{Z}^{\prime} \mathbf{T}^{\prime} & \mathbf{0} \\
\mathbf{B} \boldsymbol{\Sigma} \mathbf{A}^{\prime} & \mathbf{B} \boldsymbol{\Sigma} \mathbf{A}^{\prime} \mathbf{T}^{\prime} & \mathbf{J}
\end{array}\right]=\boldsymbol{r}\left[\begin{array}{ccc}
\mathbf{A} \boldsymbol{\Sigma} \mathbf{A}^{\prime} & \mathbf{A} \boldsymbol{\Sigma} \mathbf{A}^{\prime} \mathbf{T}^{\prime} & \mathbf{Z} \\
\mathbf{Z}^{\prime} & \mathbf{0} & \mathbf{0} \\
\mathbf{0} & \mathbf{Z}^{\prime} \mathbf{T}^{\prime} & \mathbf{0}
\end{array}\right]
$$

Then, $\operatorname{BLUP}_{\mathcal{M}}(\mathbf{u}) \in\left\{\operatorname{BLUP}_{\mathcal{T}}(\mathbf{u})\right\}$ holds.

Proof. From Lemma 2.2 (a), the equations in (2.1) and (2.4) have a common solution $\Leftrightarrow$

$$
\boldsymbol{r}\left[\begin{array}{llll}
\mathbf{Z} & \mathbf{A} \Sigma \mathbf{A}^{\prime} \mathbf{Z}^{\perp} & \mathbf{Z} & \mathbf{A} \boldsymbol{\Sigma} \mathbf{A}^{\prime} \mathbf{T}^{\prime}(\mathbf{T Z})^{\perp} \\
\mathbf{J} & \mathbf{B} \boldsymbol{\Sigma} \mathbf{A}^{\prime} \mathbf{Z}^{\perp} & \mathbf{J} & \mathbf{B} \boldsymbol{\Sigma} \mathbf{A}^{\prime} \mathbf{T}^{\prime}(\mathbf{T Z})^{\perp}
\end{array}\right]=\boldsymbol{r}\left[\begin{array}{lllll}
\mathbf{Z} & \mathbf{A} \boldsymbol{\Sigma} \mathbf{A}^{\prime} \mathbf{Z}^{\perp} & \mathbf{Z} & \mathbf{A} \boldsymbol{\Sigma} \mathbf{A}^{\prime} \mathbf{T}^{\prime}(\mathbf{T Z})^{\perp}
\end{array}\right] .
$$


(3.12) equivalently written as

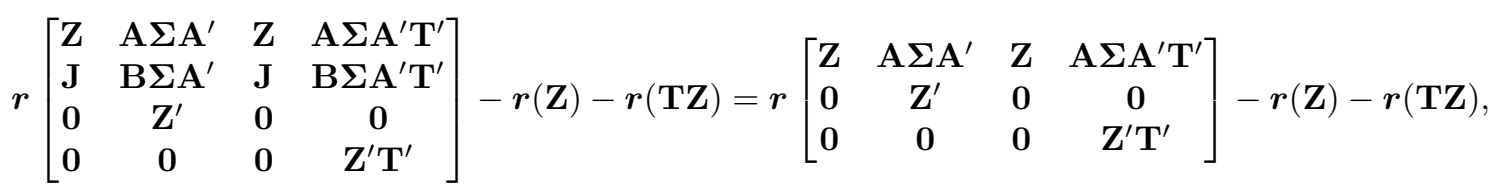

which is equivalent to (3.11).

Corollary 3.3. Let us consider $\mathcal{M}$ in (1.1) and $\mathcal{T}$ in (1.2).

(a) Let $\mathbf{J} \boldsymbol{\alpha}$ be estimable under $\mathcal{T}$ (also estimable under $\mathcal{M}$ ). Let the coefficients $\mathbf{F}_{\mathbf{J} \boldsymbol{\alpha}}$ and $\mathbf{F}_{t \mathbf{J} \boldsymbol{\alpha}}$ be as given in (2.7) and (2.8), respectively. Then the following holds.

$$
\mathbf{F}_{\mathbf{J} \boldsymbol{\alpha}} \in\left\{\mathbf{F}_{t \mathbf{J} \boldsymbol{\alpha}} \mathbf{T}\right\} \Leftrightarrow \boldsymbol{r}\left[\begin{array}{ccc}
\mathbf{A} \boldsymbol{\Sigma} \mathbf{A}^{\prime} & \mathbf{A} \boldsymbol{\Sigma} \mathbf{A}^{\prime} \mathbf{T}^{\prime} & \mathbf{Z} \\
\mathbf{Z}^{\prime} & \mathbf{0} & \mathbf{0} \\
\mathbf{0} & \mathbf{Z}^{\prime} \mathbf{T}^{\prime} & \mathbf{0} \\
\mathbf{0} & \mathbf{0} & \mathbf{J}
\end{array}\right]=\boldsymbol{r}\left[\begin{array}{ccc}
\mathbf{A} \Sigma \mathbf{A}^{\prime} & \mathbf{A} \Sigma \mathbf{A}^{\prime} \mathbf{T}^{\prime} & \mathbf{Z} \\
\mathbf{Z}^{\prime} & \mathbf{0} & \mathbf{0} \\
\mathbf{0} & \mathbf{Z}^{\prime} \mathbf{T}^{\prime} & \mathbf{0}
\end{array}\right] .
$$

In this case, $\operatorname{BLUE}_{\mathcal{M}}(\mathbf{J} \boldsymbol{\alpha}) \in\left\{\operatorname{BLUE}_{\mathcal{T}}(\mathbf{J} \boldsymbol{\alpha})\right\}$ holds.

(b) If $\mathbf{Z} \boldsymbol{\alpha}$ is estimable under the models $\mathcal{M}$ and $\mathcal{T}$ then (2.6) holds. Let the coefficients $\mathbf{F}_{\mathbf{Z} \boldsymbol{\alpha}}$ and $\mathbf{F}_{t \mathbf{z} \boldsymbol{\alpha}}$ be as given in (2.9) and (2.10), respectively. Then the following holds.

$$
\mathbf{F}_{\mathbf{Z} \boldsymbol{\alpha}} \in\left\{\mathbf{F}_{t \mathbf{Z} \boldsymbol{\alpha}} \mathbf{T}\right\} \Leftrightarrow \mathcal{C}\left[\begin{array}{cc}
\mathbf{A} \mathbf{\Sigma} \mathbf{A}^{\prime} & \mathbf{A} \boldsymbol{\Sigma} \mathbf{A}^{\prime} \mathbf{T}^{\prime} \\
\mathbf{Z}^{\prime} & \mathbf{0} \\
\mathbf{0} & \mathbf{Z}^{\prime} \mathbf{T}^{\prime}
\end{array}\right] \cap \mathcal{C}(\mathbf{Z})=\{\mathbf{0}\}
$$

In this case, $\operatorname{BLUP}_{\mathcal{M}}(\mathbf{Z} \boldsymbol{\alpha}) \in\left\{\operatorname{BLUP}_{\mathcal{T}}(\mathbf{Z} \boldsymbol{\alpha})\right\}$ holds.

\section{Funding}

There is no funding for this work.

\section{Availability of data and materials}

Not applicable.

\section{Competing interests}

The authors declare that they have no competing interests.

\section{Author's contributions}

All authors contributed equally to the writing of this paper. All authors read and approved the final manuscript.

\section{References}

[1] Alalouf, I. S., Styan, G. P. H.: Characterizations of estimability in the general linear model. Ann. Stat. 7, 194-200 (1979).

[2] Arendacká, B., Puntanen, S.: Further remarks on the connection between fixed linear model and mixed linear model. Stat. Papers. 56 (4), 1235-1247 (2015).

[3] Baksalary, J. K., Kala, S.: Linear transformations preserving best linear unbiased estimators in a general Gauss-Markoff model. Ann. Stat. 9, 913-916 (1981).

[4] Dong, B., Guo, W., Tian, Y.: On relations between BLUEs under two transformed linear models. J. Multivariate Anal. 131, 279-292 (2014). 
[5] Drygas, H.: Sufficiency and completeness in the general Gauss-Markov model. Sankhy $\bar{a}$, Ser A. 45, 88-98 (1983).

[6] Goldberger, A. S.: Best linear unbiased prediction in the generalized linear regression model. J. Amer. Statist. Assoc. 57, 369-375 (1962).

[7] Güler, N.: On relations between BLUPs under two transformed linear random-effects models. Commun. Statist. Simulation and Computation. (2020). https://doi.org/10.1080/03610918.2020.1757709

[8] Harville, D.: Extension of the Gauss-Markov theorem to include the estimation of random effects. Ann. Stat. 4, 384-395 (1976).

[9] Haslett, S. J., Puntanen, S.: Equality of BLUEs or BLUPs under two linear models using stochastic restrictions. Stat. Papers. 51 (2), 465-475 (2010).

[10] Haslett, S. J., Puntanen, S.: On the equality of the BLUPs under two linear mixed models. Metrika. 74, 381-395 (2011).

[11] Isotalo, J., Puntanen, S.: Linear prediction sufficiency for new observations in the general Gauss-Markov model. Commun. Statist. Theory and Methods. 35, 1011-1023 (2006).

[12] Liu, X., Wang, Q. W.: Equality of the BLUPs under the mixed linear model when random components and errors are correlated. J. Multivariate Anal. 116, 297-309 (2013).

[13] Marsaglia, G., Styan, G. P. H.: Equalities and inequalities for ranks of matrices, Linear Multilinear Algebra. 2, 269-292 (1974).

[14] Penrose, R.: Generalized inverse for matrices. Proc. Cambridge Philos. Soc. 51, 406-413 (1955).

[15] Puntanen, S., Styan, G. P. H. , Isotalo, J.: Matrix Tricks for Linear Statistical Models: Our Personal Top Twenty. Springer, Heidelberg (2011).

[16] Rao, C. R.: Representations of best linear unbiased estimators in the Gauss-Markoff model with a singular dispersion matrix. J. Multivariate Anal. 3, 276-292 (1973).

[17] Sun, Y., Jiang B., Jiang, H.: Computations of predictors/estimators under a linear random-effects model with parameter restrictions. Commun. Statist. Theory and Methods. 48 (14), 3482-3497 (2019).

[18] Tian, Y.: The maximal and minimal ranks of some expressions of generalized inverses of matrices. Southeast Asian Bull. Math. 25, 745-755 (2002).

[19] Tian, Y.: On equalities for BLUEs under misspecified Gauss-Markov models. Acta Mathematica Sinica. Eng. Ser. 25 (11), 1907-1920 (2009).

[20] Tian, Y.: A new derivation of BLUPs under random-effects model. Metrika. 78, 905-918 (2015).

[21] Tian, Y.: A matrix handling of predictions under a general linear random-effects model with new observations. Electron. J. Linear Algebra. 29, 30-45 (2015).

[22] Tian, Y.: Transformation approaches of linear random-effects models. Stat. Methods Appl. 26 (4), 583-608 (2017).

[23] Tian, Y., Cheng, S.: The maximal and minimal ranks of A-BXC with applications. New York J. Math. 9, 345-362 (2003).

[24] Tian, Y., Puntanen, S.: On the equivalence of estimations under a general linear model and its transformed models. Linear Algebra Appl. 430, 2622-2641 (2009).

[25] Tian, Y., Jiang, B.: An algebraic study of BLUPs under two linear random-effects models with correlated covariance matrices. Linear Multilinear Algebra. 64 (12), 2351-2367 (2016).

[26] Tian, Y., Jiang B.: Matrix rank/inertia formulas for least-squares solutions with statistical applications. Spec. Matrices. 4 (1), 130-140 (2016). 


\section{Affiliations}

MELIKE YIĞIT

ADDRESS: Sakarya University, Department of Mathematics, 54187, Sakarya, Turkey.

E-MAIL: melikeyigitt@gmail.com

ORCID ID:https://orcid.org/0000-0002-9205-7842

NESRIN GÜLER

ADDRESS: Sakarya University, Department of Econometrics, 54187, Sakarya, Turkey.

E-MAIL: nesring@sakarya.edu.tr

ORCID ID:https://orcid.org/0000-0003-3233-5377

MELEK ERIŞ BÜYÜKKAYA

AdDRESS: Karadeniz Technical University, Department of Statistics and Computer Sciences, 61080, Karadeniz Technical University.

E-MAIL: melekeris@ktu.edu.tr

ORCID ID:https://orcid.org/0000-0002-6207-5687 